\title{
Intrauterine exposure to diabetes and risk of cardiovascular disease in adolescence and early adulthood: a population-based birth cohort study
}

\author{
Laetitia Guillemette PhD, Brandy Wicklow MD, Elizabeth A.C. Sellers MD, Allison Dart MD, Garry X. Shen MD, \\ Vernon W. Dolinsky PhD, Joseph W. Gordon PhD, Davinder S. Jassal MD, Nathan Nickel PhD, \\ Todd A. Duhamel PhD, Dan Chateau PhD, Heather J. Prior MSc, Jonathan McGavock PhD
}

Cite as: CMAJ 2020 September 28;192:E1104-13. doi: 10.1503/cmaj.190797

\section{ABSTRACT \\ BACKGROUND: It is unclear whether intra- uterine exposure to maternal diabetes is associated with risk factors for cardiovas- cular disease and related end points in adulthood. We examined this potential association in a population-based birth cohort followed up to age 35 years.}

METHODS: We performed a cohort study of offspring born between 1979 and 2005 $(n=293546)$ and followed until March 2015 in Manitoba, Canada, using registry-based administrative data. The primary exposures were intrauterine exposure to gestational diabetes and type 2 diabetes mellitus. The primary outcome was a composite measure of incident cardiovascular disease events, and the secondary outcome was a composite of risk factors for cardiovascular disease in offspring followed up to age 35 years.

RESULTS: The cohort provided 3628576 person-years of data (mean age at latest follow-up 20.5 [standard deviation 6.4] years, $49.3 \%$ female); 2765 (0.9\%) of the offspring experienced a cardiovascular disease end point, and 12673 (4.3\%) experienced a cardiovascular disease risk factor. After propensity score matching, the hazard for cardiovascular disease end points was elevated in offspring exposed to gestational diabetes (adjusted hazard ratio [HR] 1.42, 95\% confidence interval [Cl] 1.12-1.79) but not type 2 diabetes (adjusted HR 1.40, 95\% Cl 0.98-2.01). A similar association was observed for cardiovascular disease risk factors (gestational diabetes: adjusted HR $1.92,95 \% \mathrm{Cl}$ 1.75-2.11; type 2 diabetes: adjusted HR $3.40,95 \% \mathrm{Cl} 3.00-3.85)$.

INTERPRETATION: Intrauterine exposure to maternal diabetes was associated with higher morbidity and risk related to cardiovascular disease among offspring up to 35 years of age.

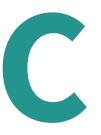

ardiovascular diseases represent the most common noncommunicable cause of death worldwide. ${ }^{1}$ Although cardiovascular disease largely affects older individuals, ${ }^{2}$ the population-level burden is evident as early as 35 years of age. ${ }^{3}$ Most adolescents in developed countries live with 1 or more risk factors for cardiovascular disease, and rates of cardiovascular disease continue to increase in individuals younger than 40 years of age. ${ }^{3}$ Lifestyle factors in childhood contribute to early-onset cardiovascular disease; however, a growing body of evidence suggests that the natural history of these problems begins in utero. ${ }^{4-6}$

Poor fetal nutrition is an established but relatively underrecognized risk factor for cardiovascular disease. ${ }^{7,8}$ Large prospective cohort studies ${ }^{9}$ and some natural experiments ${ }^{10,11}$ have shown that maternal undernutrition is associated with higher rates of premature cardiovascular disease. More recently, the early-life origins of cardiovascular disease have been linked to maternal overnutrition and hyperglycemia in pregnancy. ${ }^{12}$ In smaller birth cohorts, exposure to maternal diabetes in utero has been associated with cardiometabolic risk factor clustering in offspring in both childhood and adolescence. ${ }^{13,14}$ To date, however, these observations have not been replicated in population-based samples with prolonged offspring follow-up and a focus on harder end points related to cardiovascular disease.

Maternal diabetes is one of the most common metabolic perturbations to which fetuses may be exposed. ${ }^{15,16}$ Fetal exposure 
to maternal gestational diabetes and type 2 diabetes mellitus ${ }^{6}$ is associated with adverse cardiometabolic health in childhood, including insulin resistance, overweight, dyslipidemia, type 2 diabetes and potentially elevated blood pressure. ${ }^{17}$ For example, in a recent administrative cohort study, members of our team found that in utero exposure to diabetes during pregnancy was associated with a dose-dependent increased risk for type 2 diabetes in adolescence. ${ }^{18}$ Cohort studies from Europe have shown similar associations between in utero exposure to maternal diabetes and features of cardiometabolic disease in adolescence. ${ }^{19}$ It remains unclear, however, whether fetal exposure to maternal diabetes is associated with higher cardiovascular diseaserelated morbidity in young adulthood.

We hypothesized that intrauterine exposure to gestational diabetes or type 2 diabetes would be associated with higher cardiovascular disease morbidity relative to no intrauterine exposure to diabetes. Additionally, we hypothesized that cardiovascular disease risk would be greater following intrauterine exposure to type 2 diabetes than intrauterine exposure to gestational diabetes.

\section{Methods}

\section{Study design and data sources}

We designed an observational population-based birth cohort using administrative data held in the Manitoba Population Research Data Repository. Scrambled personal health identification numbers were deidentified by a third party (Manitoba Health, Seniors and Active Living). The scrambled identifiers allowed linkage of data from multiple databases and years of follow-up. The deidentified personal health identification numbers used for linkage are 9-digit numeric identifiers assigned by Manitoba Health, Seniors and Active Living to every person registered for health insurance in Manitoba. ${ }^{20}$

Individual data for demographic characteristics, exposures and outcomes were linked across the following data sets, described in detail elsewhere: ${ }^{21}$ hospital discharge abstracts (1979-2015), outpatient visits (as physician claims; 1979-2015), Canadian Census data (1986-2016), Diabetes Education Resource for Children and Adolescents (clinical pediatric diabetes) database (1986-2015) and Drug Program Information Network File (1995-2015). Through this process, we created a unique data set that included about $99 \%$ of all children born in the province between Apr. 1, 1979, and Mar. 31, 2005, and followed until Mar. 31, 2015.22 These methods have been described previously. ${ }^{18,21}$ Details of each database are provided in Appendix 1, Table A1-1 (available at www.cmaj.ca/lookup/ doi/10.1503/cmaj.190797/tab-related-content). For disease diagnoses, we used codes from the International Classification of Diseases, 9th Revision, Clinical Modification and the International Statistical Classification of Diseases and Related Health Problems, 10th Revision, Canadian version (for details, see Appendix 1, Additional methods and Table A1-2).

\section{Study population}

After capturing all live births during the study period, we excluded offspring with ineligible diagnoses (congenital anomaly of the cardiovascular system, endocardial fibroelastosis, congenital or familial cardiomyopathy; see Appendix 1, Table A1-2), death during the hospital stay following birth or invalid personal health identification number. We excluded both mothers and offspring with a diagnosis of diabetes other than type 2, which we defined as a diagnosis of diabetes before 7 years of age, a prescription for insulin pump supplies, a diagnosis of cystic fibrosis or a diagnosis of any other type of diabetes (see Appendix 1, Tables A1-2 and A1-3). ${ }^{6,23}$ We excluded offspring with a diagnosis of cystic fibrosis because youth with this condition are at increased risk of non-type 2-related diabetes. ${ }^{24}$ We excluded all of these conditions to limit the risk of confounding and to improve the precision of the exposure. ${ }^{25}$

\section{Exposures}

We categorized intrauterine exposure to diabetes as maternal gestational diabetes or pre-existing type 2 diabetes using established definitions. ${ }^{6,26}$ We defined gestational diabetes in mothers as a diagnosis of diabetes between 21 weeks' gestational age and 6 weeks postpartum, ${ }^{26,27}$ in the absence of a diabetes diagnosis in the previous year. We considered offspring exposed to diabetes at less than 21 weeks' gestational age to have been exposed to pre-existing type 2 diabetes. ${ }^{18}$

\section{Outcomes of interest}

The primary outcome of interest was a composite measure of the first incident cardiovascular disease end point occurring in offspring, without a viral, familial or alcoholic origin (see Appendix 1, Table A1-1 for details), between 10 years of age and the last available follow-up date. We selected the age of 10 years because our team used this cut-off in the past to distinguish between inherited or autoimmune cardiovascular disease-related risk factors and chronic, acquired cardiovascular disease-related risk factors (e.g., diabetes and hypertension). ${ }^{18,28}$ The composite outcome included diagnoses of cardiac arrest, myocardial infarction, ischemic heart disease and cerebral infarction. The secondary outcome was a composite measure of the first incident cardiovascular disease risk factor among offspring, including hypertension, dyslipidemia and type 2 diabetes (definitions in Appendix 1, Table A1-2). For offspring with multiple outcomes during the follow-up period, only the first (incident) diagnosis was considered. Because data concerning offspring outcomes were available only through 2015 , and the birth cohort was initiated in 1979, the longest period of follow-up among offspring was 35 years.

We ascertained outcomes from hospital abstracts, physician claims, the clinical pediatric diabetes database and medication prescriptions, as previously described. ${ }^{23}$ More specifically, any offspring with a prescription for a medication in a drug class listed in Appendix 1, Table A1-3, was classified by the algorithm as having a risk factor. We adapted this approach from a validated algorithm used to capture cardiovascular disease outcomes in adults within the data repository (kappa between 0.22 and 0.65 , sensitivity $54 \%-82 \%$ and specificity $82 \%-97 \%$, depending on the outcome). ${ }^{29}$ 


\section{Potential confounders}

We classified sex-adjusted birth weight as either appropriate, small for gestational age or large for gestational age. ${ }^{30}$ Birth weight less that the 10th or greater than the 90th percentile for gestational age and sex were used to classify small and large for gestational age, respectively. ${ }^{31}$ We used the lambda-musigma method to calculate $z$ scores from birth weight derived from population-based references according to the Fenton method..$^{30}$ We defined preterm birth as live birth at less than 37 weeks' gestation. ${ }^{32}$ We defined residence using postal code at birth; residence outside the 2 major cities in Manitoba was classified as rural. We defined socioeconomic status at birth using the Socioeconomic Factor Index, version 2 (SEFI-2). ${ }^{33,34} \mathrm{~A}$ score of 0 represents the provincial average, and $95 \%$ of scores fall within 2 points above or below the mean, with positive values indicating higher deprivation (i.e., lower socioeconomic status) and negative values indicating lower deprivation. We also adjusted for year of birth, sex and maternal age at delivery, as derived from hospital abstracts.

\section{Statistical analysis}

We used descriptive statistics to summarize the characteristics of the cohort. We used analysis of variance and $\chi^{2}$ tests to test for differences in baseline characteristics between groups. We used Cox proportional hazards regression models to generate adjusted hazard ratios (HRs) and 95\% confidence intervals (Cls) for the composite outcomes in offspring, treating mortality as a competing risk. We used the Fine-Gray method to account for the competing risk of mortality. ${ }^{35}$ We tested the proportional hazards assumption by examining the correlation between Schoenfeld residuals and terms for time, log of time and time squared and then visually checking proportionality by plotting stratified lognegative-log curves; no significant violations were detected. We censored individuals who died or outmigrated either before reaching 10 years of age or, among those aged 10 or older, before experiencing an outcome. We included an interaction term for exposure to maternal diabetes and offspring sex in the models, because the cardiovascular disease literature indicates possible differences between males and females. ${ }^{36}$ To control for possible clustering associated with hospital of birth, we added hospital of birth as a categorical variable to the models described above. To account for possible clustering effects within families, we conducted a marginal Cox model that used a covariance matrix estimate to account for dependence of intracluster effects within families, and included propensity score matching and a categorical variable for hospital of birth. This analysis would account for possible clustering of outcomes within hospitals and families.

We ran 2 logistic regression models to predict exposure to maternal diabetes, comparing children exposed to gestational diabetes in utero with children not exposed to any diabetes in utero, and also comparing children exposed to type 2 diabetes in utero with children not exposed to any diabetes in utero. The covariables were birth weight, sex, preterm birth $(<37 \mathrm{wk}$ gestation), large for gestational age, small for gestational age, year of birth, mother's age at birth, SEFI-2, and urban or rural residence of the mother at the time of birth. From these regression models, we generated predicted probabilities of exposure to diabetes and used them to match children exposed to either gestational diabetes or type 2 diabetes with unexposed controls on the basis of propensity scores (at a ratio of $1: 10$ ), with a limit of the control propensity score being within 0.01 of the diabetes case propensity score. We calculated standardized differences before and after matching. We employed Cox proportional hazards regression models to estimate the time to the cardiovascular disease composite measure and the cardiovascular disease risk composite measure, controlling for diabetes exposure for the matched groups.

Statistical significance was set at 0.05 for 2-sided tests. We performed all analyses with SAS 9.4 software (SAS Institute Inc.) and produced the cumulative incidence curves with $\mathrm{R}$ software.

We imputed data for all missing gestational ages and birth weights by applying the Markov chain Monte Carlo imputation method with a single chain. The variables used in the imputation models were birth year, mother's age, sex, Apgar score at 5 minutes, region of residence and SEFI-2. We performed sensitivity analyses, using different approaches to account for 2 possible sources of clustering: within families and within hospitals. To account for clustering within families, we repeated the analyses described above with a randomly selected sample consisting of $80 \%$ of the mother-infant pairs, but restricted to 1 child per mother, to determine whether the point estimates changed when data were restricted to a single offspring. To account for hospital-level clustering, we repeated the analyses described above with adjustment for the hospital where offspring were delivered. We added hospital of birth to the propensity score-matched models as a categorical variable. To test for possible sex-specific differences in the associations that we observed, we repeated all propensity score-matched analyses, accounting for competing risks, with the addition of an interaction term to test for an interaction between offspring sex and exposure to maternal diabetes.

\section{Ethics approval}

This study was approved by the Health Research Ethics Board at the University of Manitoba (HREB HS19742), in accordance with the Declaration of Helsinki and the provincial Health Information Privacy Committee (HIPC 2016/2017-06).

\section{Results}

A total of 359919 births from 200754 mothers (with valid insurance) were identified during the study period. After exclusion of those who did not meet the inclusion criteria, 293546 offspring from 189939 mothers were included in the birth cohort and provided person-years of follow-up (Figure 1). Most (282 119 or $96.1 \%$ ) of these offspring were not exposed to maternal diabetes in utero, whereas 8210 (2.8\%) were exposed to gestational diabetes and $3217(1.1 \%)$ to pre-existing type 2 diabetes (Table 1 ). The mean age of offspring at latest follow-up was 20.5 (standard deviation [SD] 6.4) years (range 10-35 yr).

Intrauterine exposure to both gestational diabetes and type 2 diabetes became more common over the study period $(p<0.001$; 


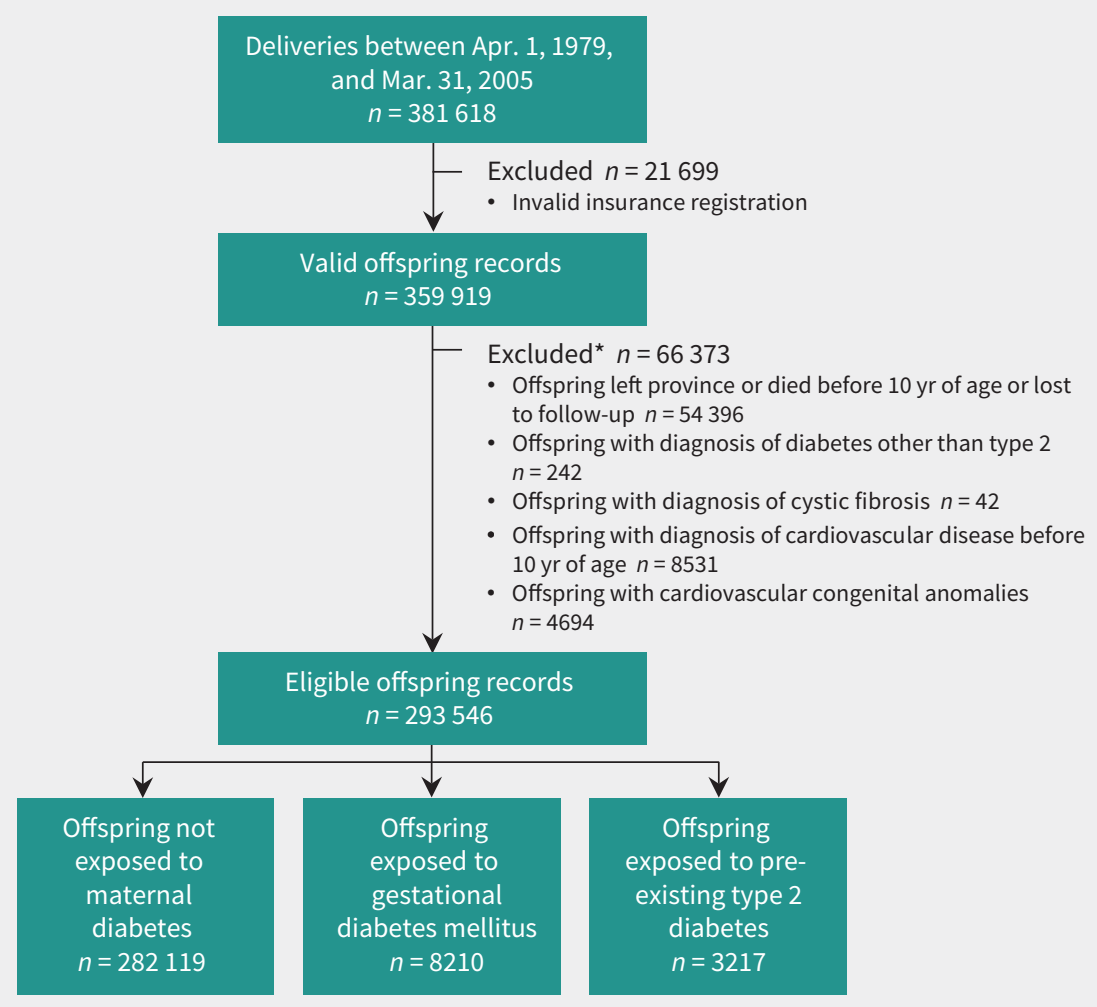

Figure 1: Study flow diagram showing derivation of the cohort of offspring used to examine the association between intrauterine exposure to diabetes and risk of cardiovascular disease. "Reasons for exclusion are not mutually exclusive.

Table 1). Offspring exposed to diabetes in utero were more likely to be born to older mothers, to be born large for gestational age, to be born preterm, and to reside in a rural area or in a household with greater socioeconomic deprivation (Table 1).

During the follow-up period, 15438 (5.3\%) of the cohort experienced either an event or a risk factor related to cardiovascular disease. The primary outcome, the first incident cardiovascular disease end point, was observed in $2765(0.9 \%)$ of the cohort, and the secondary outcome, the first incident cardiovascular disease risk factor, was observed in 12673 (4.3\%) of the cohort. The 3 most frequent diagnoses among the offspring were hypertension ( $n=8713)$, type 2 diabetes $(n=3568)$ and ischemic heart disease $(n=715)$.

The crude incidence of the primary outcome increased across exposure strata (not exposed, 0.76 per 1000/yr; exposed to gestational diabetes, 0.90 per 1000/yr; exposed to type 2 diabetes, 1.04 per 1000/yr; Table 2). Most offspring experienced a cardiovascular disease risk factor without experiencing any related end points, and few reached a cardiovascular disease end point before diagnosis of a relevant risk factor. Crude rates of individual outcomes and risk factors are presented in Appendix 1, Tables A1-4 and A1-5, respectively. The mean age at occurrence of the first cardiovascular disease risk factor was significantly lower in offspring exposed to diabetes in utero (not exposed, 21.2 [SD 5.9] yr; exposed to gestational diabetes, 19.5 [SD 5.8] yr; exposed to type 2 diabetes, 17.3 [SD 5.2] yr; $p<0.001$; Table 2).

Adjusted Cox proportional hazards regression models showed that exposure to diabetes in utero was associated with higher risk of the primary composite outcome (gestational diabetes v. not exposed: adjusted HR 1.27, 95\% $\mathrm{Cl} 1.02-1.59$; type 2 diabetes v. not exposed: adjusted HR 1.48, 95\% Cl 1.05-2.07) (Figure 2A). Similar associations were seen for the secondary outcome (gestational diabetes v. not exposed: adjusted HR 1.85, 95\% Cl 1.69-2.02; type 2 diabetes v. not exposed: adjusted HR 3.38, 95\% Cl 3.02-3.77) ( Figure 2B).

Propensity scores were calculated from the main confounding variables for the 2 main exposures, and distributions are presented in Appendix 1, Figure A1-1. Using propensity scores for the 8210 offspring exposed to gestational diabetes, 8208 $(100.0 \%)$ had at least 1 match and $7111(86.6 \%)$ had 10 matches in the unexposed group. Similarly, 3212 (99.8\%) of the 3217 offspring exposed to type 2 diabetes had at least 1 match and 2781 $(86.4 \%)$ had 10 matches in the unexposed group. The standardized differences between the matched groups were all below 
Table 1: Characteristics of the cohort of offspring (born 1979-2005), according to exposure to maternal diabetes in utero

\begin{tabular}{|c|c|c|c|c|c|}
\hline \multirow[b]{2}{*}{ Characteristic } & \multicolumn{3}{|c|}{ Exposure group; no. (\%) of offspring* } & \multicolumn{2}{|c|}{ Comparison; standardized difference } \\
\hline & $\begin{array}{c}\text { Not exposed } \\
n=282119\end{array}$ & $\begin{array}{l}\text { Exposed to GDM } \\
n=8210\end{array}$ & $\begin{array}{c}\text { Exposed to T2D } \\
n=3217\end{array}$ & $\begin{array}{l}\text { Maternal GDM v. } \\
\text { no exposure }\end{array}$ & $\begin{array}{c}\text { Maternal T2D v. } \\
\text { no exposure }\end{array}$ \\
\hline $\begin{array}{l}\text { Age of mother at birth, } y r \text {, } \\
\text { mean } \pm \text { SD }\end{array}$ & $27.0 \pm 5.5$ & $28.5 \pm 6.1$ & $29.5 \pm 5.6$ & 0.25 & 0.45 \\
\hline Birth weight, g, mean \pm SD & $3463.7 \pm 557.0$ & $3616.1 \pm 615.7$ & $3515.6 \pm 670.4$ & 0.26 & 0.08 \\
\hline Large for gestational age & $22806(8.1)$ & $1973(24.0)$ & $928(28.8)$ & 0.45 & 0.56 \\
\hline Small for gestational age & $24201(8.6)$ & $375(4.6)$ & $160(5.0)$ & 0.16 & 0.14 \\
\hline Preterm birth $\uparrow$ & $16014(5.7)$ & $715(8.7)$ & $606(18.8)$ & 0.12 & 0.41 \\
\hline Female sex & $139223(49.3)$ & $3894(47.4)$ & $1559(48.5)$ & 0.04 & 0.02 \\
\hline Urban residence $\ddagger$ & $158527(56.2)$ & $4213(51.3)$ & $1402(43.6)$ & 0.10 & 0.25 \\
\hline \multicolumn{6}{|l|}{ Birth year§ } \\
\hline 1979-1984 & $43641(15.5)$ & $489(6.0)$ & $157(4.9)$ & 0.31 & 0.36 \\
\hline $1985-1989$ & $63655(22.6)$ & $1808(22.0)$ & $643(20.0)$ & 0.01 & 0.06 \\
\hline 1990-1994 & $64122(22.7)$ & 2095 (25.5) & $742(23.1)$ & 0.07 & 0.008 \\
\hline 1995-1999 & $57547(20.4)$ & $1713(20.9)$ & $774(24.1)$ & 0.01 & 0.09 \\
\hline $2000-2005$ & $53154(18.8)$ & $2105(25.6)$ & $901(28.0)$ & 0.16 & 0.22 \\
\hline SEFI-2 score, mean \pm SD & $0.11 \pm 1.11$ & $0.56 \pm 1.23$ & $0.74 \pm 1.23$ & 0.38 & 0.53 \\
\hline
\end{tabular}

Table 2: Crude rates of cardiovascular disease end points and risk factors among offspring exposed to maternal diabetes in utero and those not exposed to maternal diabetes

\begin{tabular}{|c|c|c|c|}
\hline End point or risk factor & $\begin{array}{l}\text { Not exposed } \\
\text { to diabetes } \\
n=282119\end{array}$ & $\begin{array}{c}\text { Exposed to } \\
\text { gestational diabetes } \\
\qquad=8210\end{array}$ & $\begin{array}{c}\text { Exposed to } \\
\text { type } 2 \text { diabetes mellitus } \\
n=3217\end{array}$ \\
\hline Any outcome/no. of PYs & $14464 / 3505787$ & $610 / 90059$ & $364 / 32730$ \\
\hline Incidence per 1000/yr & 4.18 & 6.77 & 11.12 \\
\hline \multicolumn{4}{|l|}{ CVD events } \\
\hline Cases of CVD end point/no. PYs & $2650 / 3508792$ & $81 / 90098$ & $34 / 32744$ \\
\hline Incidence per 1000/yr & 0.76 & 0.90 & 1.04 \\
\hline Age at first event, yr, mean \pm SD & $21.3 \pm 5.6$ & $19.7 \pm 5.4$ & $20.1 \pm 5.0$ \\
\hline \multicolumn{4}{|l|}{ CVD risk factors } \\
\hline Cases of CVD risk factor/no. PYs & 11814 / 3514196 & $529 / 90285$ & $330 / 32690$ \\
\hline Incidence per 1000/yr & 3.36 & 5.86 & 10.09 \\
\hline Age at first risk factor, yr, mean \pm SD & $21.2 \pm 5.9$ & $19.5 \pm 5.8$ & $17.3 \pm 5.2$ \\
\hline
\end{tabular}

0.10 (Appendix 1, Figure A1-2), which indicates balance between the cases and controls. When hazards were calculated on the matched samples and adjusted for competing risks, the effect sizes and precision of the estimates remained similar for the association between exposure to maternal diabetes in utero and incident cardiovascular disease risk factors (Figure 3). The only association that was no longer statistically significant after propensity score matching was between exposure to maternal type 2 diabetes and cardiovascular disease-related outcomes (HR 1.40, 95\% Cl 0.98-2.01).

Exposure to type 2 diabetes in utero was associated with a higher risk for pooled primary and secondary outcomes 
A

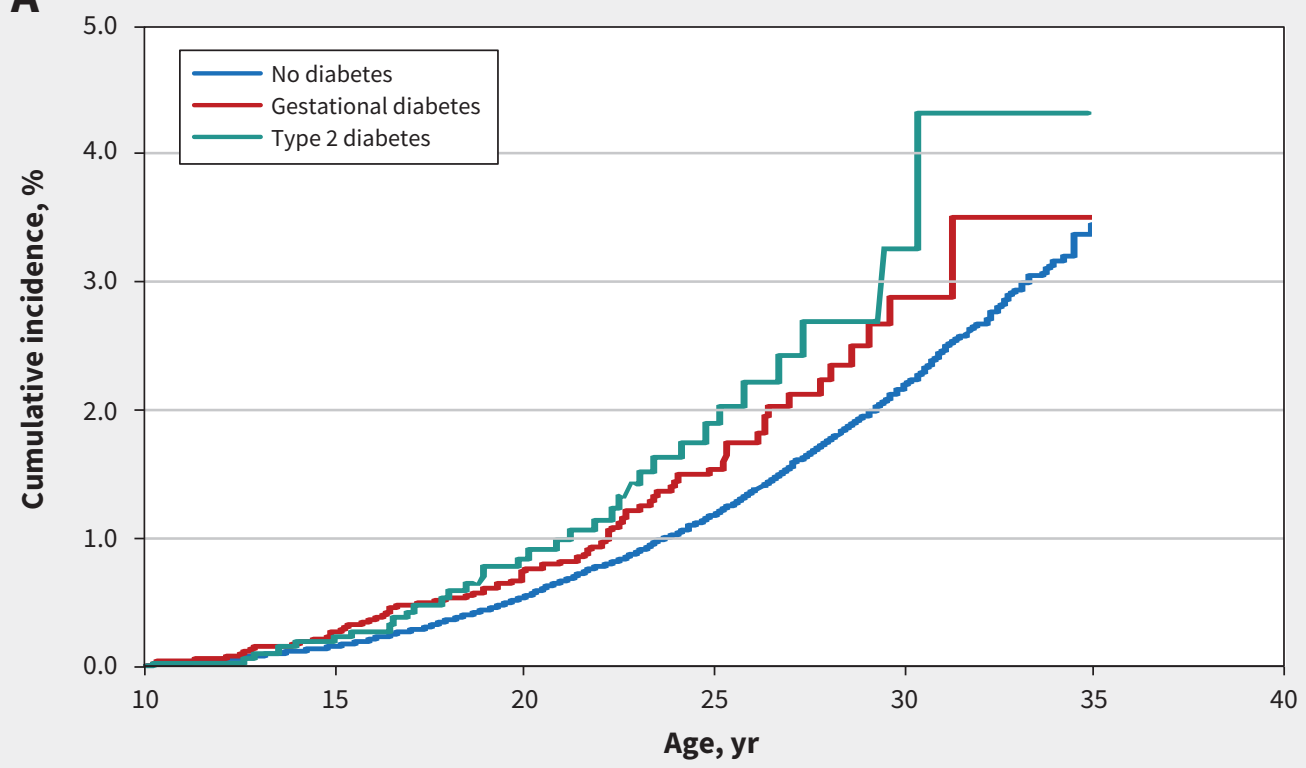

No. at risk

$\begin{array}{lrrrrrr}\text { No diabetes } & 282119 & 221054 & 158482 & 89022 & 31598 & 1175 \\ \text { GDM } & 8210 & 5901 & 4082 & 1961 & 360 & 6 \\ \text { T2D } & 3217 & 2271 & 1444 & 684 & 121 & 0\end{array}$

B

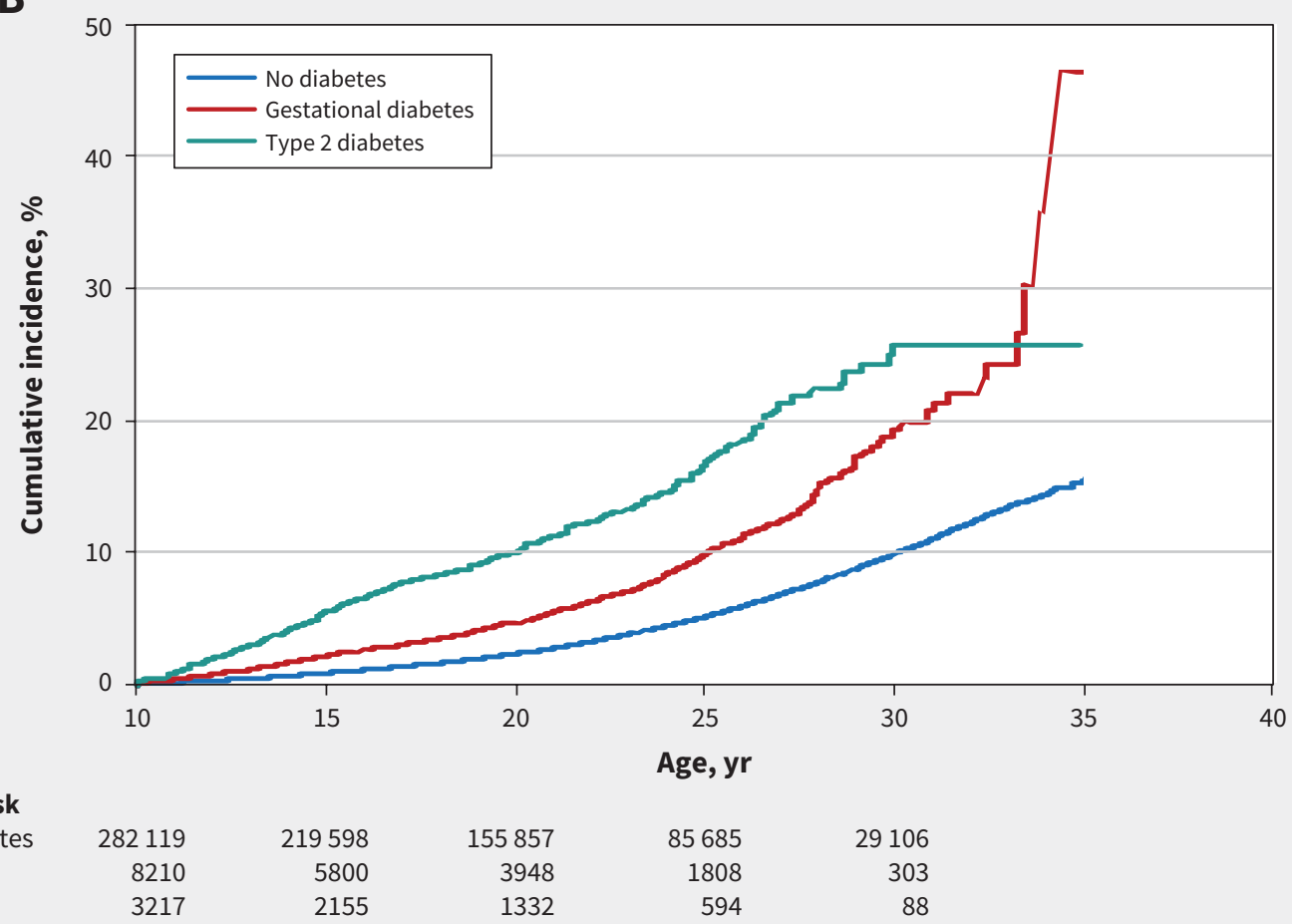

Figure 2: Adjusted cumulative incidence curves for the risk of cardiovascular disease, according to maternal diabetes exposure, in relation to offspring age, in years, with adjustment for the following factors: maternal age at offspring birth; Socioeconomic Factor Index, version 2; rural residence; birth weight; small for gestational age; large for gestational age; and preterm birth. The number of offspring at risk at each time point, beginning at 10 years of age (start of the "at-risk" period) is shown below each panel. A) Offspring reaching a cardiovascular disease end point only. B) Offspring reaching a cardiovascular disease risk factor only. In Figure $2 \mathrm{~B}$, numbers at 35 years of age were censored, because some were too small to be reported. Note: $\mathrm{GDM}=$ gestational diabetes mellitus, $\mathrm{T} 2 \mathrm{D}=$ type 2 diabetes mellitus. 


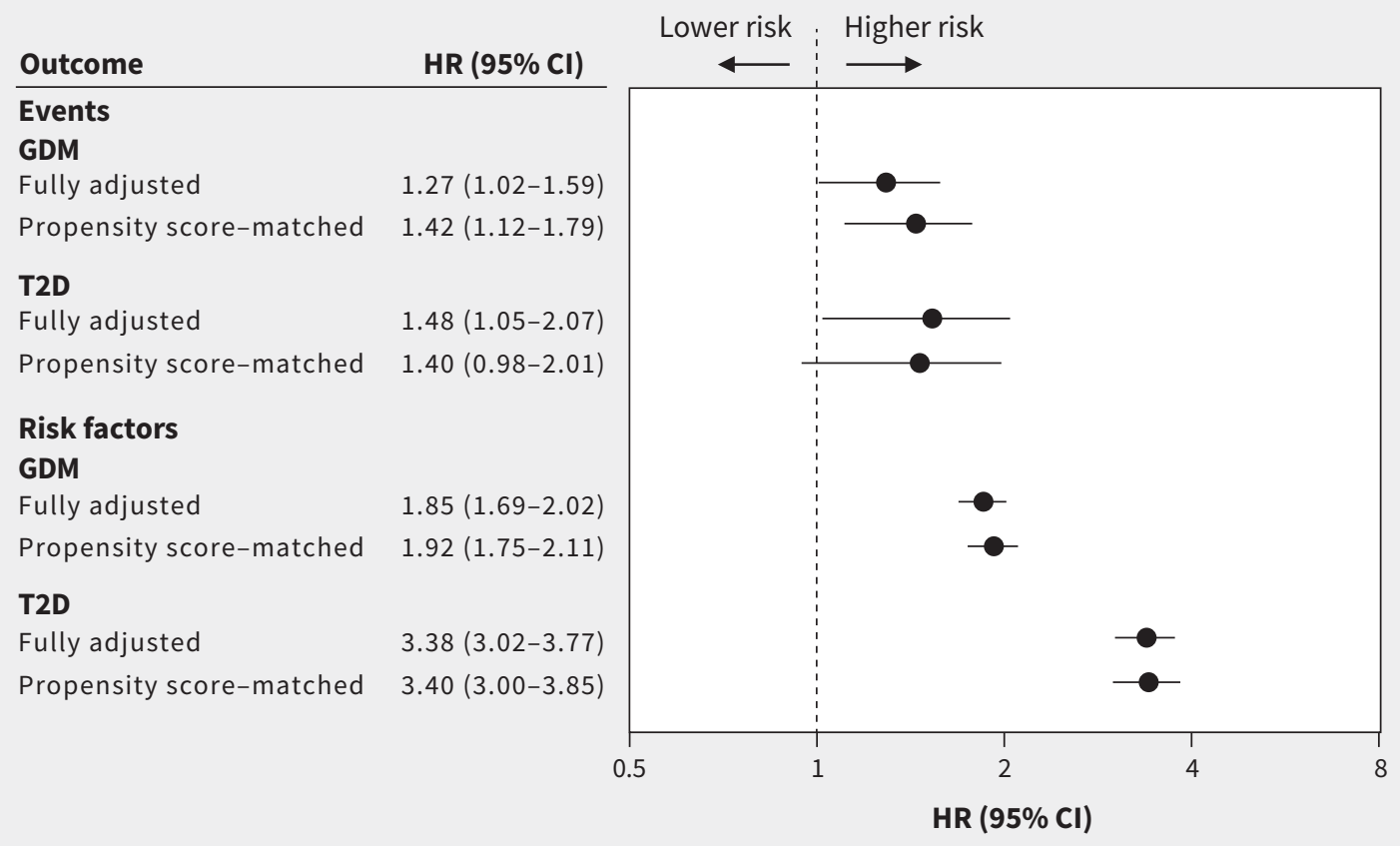

Figure 3: Cox proportional hazards for cardiovascular disease-related events and risk factors before and after propensity score matching. Note: $\mathrm{GDM}=$ gestational diabetes mellitus, HR = hazard ratio, T2D = type 2 diabetes mellitus. "Fully adjusted" means adjusted for the following factors: maternal age at offspring birth; Socioeconomic Factor Index, version 2; rural residence; birth weight; small for gestational age; large for gestational age; and preterm birth.

compared with exposure to gestational diabetes. Pooling outcomes showed a $1.65(95 \% \mathrm{Cl} 1.52-1.79)$ and $2.74(95 \% \mathrm{Cl}$ 4.26-3.05) increased risk for a cardiovascular disease-related outcome following exposure to gestational diabetes and type 2 diabetes in utero, respectively (Appendix 1, Table A1-6). These associations remained significant after the outcome of type 2 diabetes among offspring was excluded from the analyses. In sensitivity analyses, when risk factors were assessed individually, the hazard for hypertension and type 2 diabetes among offspring remained significantly higher for those exposed to gestational diabetes and type 2 diabetes in utero (Appendix 1, Table A1-7).

When the data were restricted to a subsample of randomly selected offspring, restricted to 1 per mother $(n=155720)$, and competing risks were accounted for, the strength of the associations and statistical significance remained similar to those of the primary analyses (Table 3). Similarly, when analyses were repeated and adjusted for hospital of birth, the strength of the associations remained similar (Table 3). Marginal Cox models accounting for intrafamily clustering, with adjustment for all covariables, hospital of birth and competing risks, showed nearly identical point estimates, and the associations presented above remained statistically significant (Table 3). When propensity score-matched analyses accounting for competing risks were repeated, with exclusion of cerebral infarction from the composite outcome, exposure to gestational diabetes was associated with cardiovascular disease events (HR 1.35, 95\% Cl 1.02-179), but exposure to maternal type 2 diabetes was not (HR 1.09, 95\% Cl 0.68-1.74). When the interaction term for offspring sex and exposure to maternal diabetes was added to the models presented in Table 3, no significant interactions were observed in any model.

The results for male and female offspring separately are presented in Appendix 1, Table A1-8. When these models were repeated to assess the possibility of sex differences in these results, we did not observe a significant interaction between sex and exposure to maternal diabetes.

\section{Interpretation}

In this population-based administrative birth cohort study, fetal exposure to maternal gestational diabetes or type 2 diabetes, respectively, was associated with a 1.27 -fold and 1.48 -fold higher risk for cardiovascular disease morbidity and a 1.85 -fold and 3.38-fold higher risk for cardiovascular disease risk factors before 35 years of age, compared with not being exposed to diabetes in utero. Furthermore, the first cardiovascular disease-related outcome occurred 2 to 4 years earlier among offspring exposed to either gestational diabetes or type 2 diabetes in utero. The higher cardiovascular disease morbidity among offspring exposed to diabetes in utero appeared to be driven by earlyonset type 2 diabetes and hypertension. These observations 
Table 3: Sensitivity analyses testing for associations between maternal diabetes exposure and cardiovascular disease in offspring, with additional adjustment for confounding

Exposure; adjusted HR (95\% CI)

End point or risk factor

Maternal gestational diabetes v. no diabetes exposure
Maternal type 2 diabetes $v$. no diabetes exposure

Single births, fully adjusted*

CVD end points

$1.38(1.00-1.91)$

$1.68(1.02-2.76)$

CVD risk factors

$1.58(1.37-1.83)$

$3.44(2.89-4.11)$

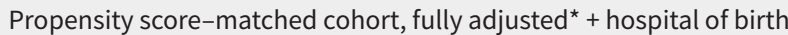

CVD end points

$1.42(1.20-1.79)$

$1.39(0.97-1.98)$

CVD risk factors

$1.90(1.73-2.08)$

$3.28(2.90-3.71)$

Marginal Cox model, $†$ fully adjusted ${ }^{*}+$ hospital of birth

CVD end points

$1.42(1.12-1.80)$

$1.39(0.96-1.99)$

CVD risk factors

$1.90(1.72-2.09)$

Note: $\mathrm{Cl}=$ confidence interval, $\mathrm{CVD}=$ cardiovascular disease, $\mathrm{HR}=$ hazard ratio

* "Fully adjusted" means adjusted for the following factors: maternal age at offspring birth; Socioeconomic Factor Index, version 2; rural residence; birth weight; small for gestational age; large for gestational age; and preterm birth.

†Marginal Cox modelling accounts for intrafamily clustering.

support our hypothesis that cardiovascular disease morbidity in adolescence and early adulthood is related to exposure to maternal diabetes in utero.

The number of children exposed in utero to gestational diabetes and type 2 diabetes has increased over the past few decades. ${ }^{16,37}$ Population-based studies by our group and smaller cross-sectional studies of children 5 to 18 years old have shown a higher prevalence of type 2 diabetes, altered glucose metabolism, higher blood pressure and arterial stiffness among offspring exposed to maternal gestational diabetes ${ }^{6,38-43}$ relative to unexposed offspring. We have extended those findings by showing that cardiovascular disease morbidity was increased and that the time to a first cardiovascular disease risk factor was reduced following fetal exposure to gestational diabetes, effects that were amplified following exposure to type 2 diabetes in utero. While it is fairly well established that intrauterine exposure to diabetes is associated with a greater risk for type 2 diabetes in offspring, we report that it is also associated with incident hypertension and myocardial infarction in young adulthood, which further reinforces the concept that intrauterine exposure to an adverse metabolic milieu predisposes offspring to chronic diseases into adulthood.

\section{Limitations}

As with all studies using administrative databases, there was a risk of misclassification bias through the use of data not collected specifically for research purposes. We tried to reduce this bias by triangulating exposures and outcomes across multiple databases ${ }^{3,6}$ with previously used algorithms. This approach circumvented the problem of modified criteria for gestational diabetes during the study period and misdiagnosis of type 2 diabetes as gestational diabetes. ${ }^{6,26}$
The precision of our outcomes of interest was limited because we relied on algorithms that have been validated in adults, but not in pediatric populations. To increase the precision of our estimates, we restricted outcomes to those acquired after 10 years of age, as we have done previously. These algorithms had modest precision, with relatively low kappa values, and therefore were at risk of misclassifying some individuals. Also, this precludes the possibility that the cause of the cardiovascular disease-related outcomes was an autoimmune condition or was related to congenital factors. Notably, only the definition of pediatric diabetes has been validated in the registry that we used. ${ }^{23}$

We had no access to clinical or lifestyle details (such as body mass index, blood chemistry, diet, physical activity patterns or smoking rates) that are known to affect the risk of cardiovascular disease and that could mediate or confound part of the observed relations. Additionally, various maternal factors, including diabetes control in pregnancy and Indigenous status, were not available for these analyses.

\section{Conclusion}

In this population-level birth cohort study, offspring exposed to gestational diabetes or type 2 diabetes in utero had a twoto three-fold higher risk for and earlier onset of cardiovascular disease-related morbidity in the first 35 years of life. This risk was principally driven by higher rates of hypertension, type 2 diabetes and ischemic heart disease among those exposed to diabetes in utero. Future studies should endeavour to replicate these findings in other settings and extend follow-up of similar cohorts into older age to assess whether the relative contribution of intrauterine diabetes exposure to cardiovascular disease-related morbidity remains stable over time. Screening children with in utero exposure to diabetes for cardiovascular 
disease risk factors might help to evaluate the future burden related to cardiovascular disease in the population.

\section{References}

1. McAloon CJ, Boylan LM, Hamborg T, et al. The changing face of cardiovascular disease 2000-2012: an analysis of the World Health Organisation global health estimates data. Int J Cardiol 2016;224:256-64.

2. Rich MW, Chyun DA, Skolnick AH, et al. Knowledge gaps in cardiovascular care of older adults: a scientific statement from the American Heart Association, American College of Cardiology, and American Geriatrics Society: executive summary. J Am Geriatr Soc 2016;64:2185-92.

3. Go AS, Mozaffarian D, Roger VL, et al. Heart disease and stroke statistics-2013 update: a report from the American Heart Association. Circulation 2013;127:e6-e245.

4. Barker DJ, Osmond C, Golding J, et al. Growth in utero, blood pressure in childhood and adult life, and mortality from cardiovascular disease. BMJ 1989;298:564-7.

5. Robinson S, Walton RJ, Clark PM, et al. The relation of fetal growth to plasma glucose in young men. Diabetologia 1992;35:444-6.

6. Sellers EA, Dean HJ, Shafer LA, et al. Exposure to gestational diabetes mellitus: impact on the development of early-onset type 2 diabetes in Canadian First Nations and non-First Nations offspring. Diabetes Care 2016;39:2240-6.

7. Tian J, Qiu M, Li Y, et al. Contribution of birth weight and adult waist circumference to cardiovascular disease risk in a longitudinal study. Sci Rep 2017;7:9768.

8. Lawlor DA, Ronalds $\mathrm{G}$, Clark H, et al. Birth weight is inversely associated with incident coronary heart disease and stroke among individuals born in the 1950s: findings from the Aberdeen children of the 1950s prospective cohort study. Circulation 2005;112:1414-8.

9. Rich-Edwards JW, Kleinman K, Michels KB, et al. Longitudinal study of birth weight and adult body mass index in predicting risk of coronary heart disease and stroke in women. BMJ 2005;330:1115.

10. Roseboom TJ, van der Meulen JH, Osmond C, et al. Coronary heart disease after prenatal exposure to the Dutch famine, 1944-45. Heart 2000;84:595-8.

11. Sparén $P$, Vagero $D$, Shestov $D B$, et al. Long-term mortality after severe starvation during the siege of Leningrad: prospective cohort study. BMJ 2004;328:11.

12. Fleming TP, Watkins AJ, Velazquez MA, et al. Origins of lifetime health around the time of conception: causes and consequences. Lancet 2018;391:1842-52.

13. Hochner H, Friedlander Y, Calderon-Margalit R, et al. Associations of maternal prepregnancy body mass index and gestational weight gain with adult offspring cardiometabolic risk factors: the Jerusalem Perinatal Family Follow-up Study. Circulation 2012;125:1381-9.

14. Warrington NM, Beaumont RN, Horikoshi M, et al. Maternal and fetal genetic effects on birth weight and their relevance to cardio-metabolic risk factors. Nat Genet 2019;51:804-14.

15. Facts and figures from a public health perspective. Ottawa: Public Health Agency of Canada; 2011.

16. Lawrence JM, Contreras R, Chen W, et al. Trends in the prevalence of preexisting diabetes and gestational diabetes mellitus among a racially/ethnically diverse population of pregnant women, 1999-2005. Diabetes Care 2008;31:899-904.

17. Huang RC, Prescott SL, Godfrey KM, et al. Assessment of cardiometabolic risk in children in population studies: underpinning developmental origins of health and disease mother-offspring cohort studies. J Nutr Sci 2015;4:e12.

18. Wicklow BA, Sellers EAC, Sharma AK, et al. Association of gestational diabetes and type 2 diabetes exposure in utero with the development of type 2 diabetes in First Nations and non-First Nations offspring. JAMA Pediatr 2018;172:724-31.

19. Yu Y, Arah OA, Liew Z, et al. Maternal diabetes during pregnancy and early onset of cardiovascular disease in offspring: population based cohort study with 40 years of follow-up. BMJ 2019 Dec 4;367:16398. doi: 10.1136/bmj.l6398.

20. Nickel NC, Chateau DG, Martens PJ, et al. Data resource profile: Pathways to Health and Social Equity for Children (PATHS Equity for Children). Int $\mathrm{J}$ Epidemiol 2014;43:1438-49.
21. Heaman MI, Martens PJ, Brownell MD, et al. The association of inadequate and intensive prenatal care with maternal, fetal, and infant outcomes: a populationbased study in Manitoba, Canada. J Obstet Gynaecol Can 2019;41:947-59.

22. Heaman M, Kingston D, Helewa ME, et al. Perinatal services and outcomes in Manitoba. Winnipeg: Manitoba Centre for Health Policy; 2012.

23. Dart AB, Martens PJ, Sellers EA, et al. Validation of a pediatric diabetes case definition using administrative health data in Manitoba, Canada. Diabetes Care 2011;34:898-903.

24. Ode KL, Moran A. New insights into cystic fibrosis-related diabetes in children Lancet Diabetes Endocrinol 2013;1:52-8.

25. Ornoy A, Reece EA, Pavlinkova G, et al. Effect of maternal diabetes on the embryo, fetus, and children: congenital anomalies, genetic and epigenetic changes and developmental outcomes. Birth Defects Res C Embryo Today 2015;105:53-72.

26. Shen GX, Shafer LA, Martens PJ, et al. Does First Nations ancestry modify the association between gestational diabetes and subsequent diabetes: a historical prospective cohort study among women in Manitoba, Canada. Diabet Med 2016;33:1245-52.

27. Dart AB, Ruth CA, Sellers EA, et al. Maternal diabetes mellitus and congenital anomalies of the kidney and urinary tract (CAKUT) in the child. Am J Kidney Dis 2015;65:684-91.

28. Dart A, Lavallee B, Chartrand C, et al. Screening for kidney disease in Indigenous Canadian children: the FINISHED screen, triage and treat program. Paediatr Child Health 2018;23:e134-42.

29. Lix L, Yogendran M, Mann J. Defining and validating chronic diseases: an administrative data approach - an update with ICD-10-CA2006. Winnipeg: University of Manitoba, Manitoba Centre for Health Policy; 2008.

30. Fenton TR, Kim JH. A systematic review and meta-analysis to revise the Fenton growth chart for preterm infants. BMC Pediatr 2013;13:59.

31. Kramer MS, Platt RW, Wen SW, et al. A new and improved population-based Canadian reference for birth weight for gestational age. Pediatrics 2001;108:E35.

32. Preterm birth. Geneva: World Health Organization; 2017.

33. Metge $\mathrm{C}$, Chateau $\mathrm{D}$, Prior $\mathrm{H}$, et al. Composite measures/indices of health and health system performance. Winnipeg: University of Manitoba, Faculty of Medicine, Department of Community Health Sciences, Manitoba Centre for Health Policy; 2009.

34. Term: socioeconomic factor index (SEFI) - version 2 (SEFI-2) [glossary definition]. Winnipeg: Manitoba Centre for Health Policy; 2016.

35. Austin PC, Fine JP. Practical recommendations for reporting Fine-Gray model analyses for competing risk data. Stat Med 2017;36:4391-400.

36. Miller VM. Sex-based differences in vascular function. Womens Health (Lond) 2010;6:737-52.

37. Beckles GL, Zhu J, Moonesinghe R; Centers for Disease Control and Prevention. Diabetes - United States, 2004 and 2008. MMWR Supp/ 2011;60:90-3.

38. Aceti A, Santhakumaran S, Logan KM, et al. The diabetic pregnancy and offspring blood pressure in childhood: a systematic review and meta-analysis. Diabetologia 2012;55:3114-27.

39. Lee H, Jang HC, Park HK, et al. Early manifestation of cardiovascular disease risk factors in offspring of mothers with previous history of gestational diabetes mellitus. Diabetes Res Clin Pract 2007;78:238-45.

40. Ma RCW, Tutino GE, Lillycrop KA, et al. Maternal diabetes, gestational diabetes and the role of epigenetics in their long-term effects on offspring. Prog Biophys Mol Biol 2015;118:55-68

41. Malcolm J. Through the looking glass: gestational diabetes as a predictor of maternal and offspring long-term health. Diabetes Metab Res Rev 2012;28:307-11.

42. Tam WH, Ma RC, Yang X, et al. Glucose intolerance and cardiometabolic risk in children exposed to maternal gestational diabetes mellitus in utero. Pediatrics 2008;122:1229-34.

43. Tam WH, Ma RC, Yip GW, et al. The association between in utero hyperinsulinemia and adolescent arterial stiffness. Diabetes Res Clin Pract 2012;95:169-75. 
Competing interests: Brandy Wicklow is currently the site principal investigator for a Boehringer Ingelheim study unrelated to the current study content. No other competing interests were declared.

This article has been peer reviewed.

Affiliations: Children's Hospital Research Institute of Manitoba (Guillemette, Wicklow, Sellers, Dart, Shen, Dolinsky, Gordon, McGavock); Developmental Origins of Health and Disease Research Network (DEVOTION) (Guillemette, Nickel, McGavock) and Diabetes Research Envisioned and Accomplished in Manitoba (DREAM) Theme (Wicklow, Sellers, Dart, Dolinsky, Gordon, McGavock), Children's Hospital Research Institute of Manitoba; Rady Faculty of Health Sciences (Shen), Section of Cardiology, Department of Internal Medicine, Max Rady College of Medicine, Rady Faculty of Health Sciences (Jassal), Department of Community Health Sciences (Chateau), Health, Leisure and Human Performance Research Institute, Faculty of Kinesiology and Recreation Management (Duhamel), University of Manitoba; Manitoba Centre for Health Policy (Nickel, Chateau, Prior), Winnipeg, Man.

Contributors: Laetitia Guillemette contributed to study conception, collected data, helped with the analyses and drafted the initial manuscript. Brandy Wicklow, Elizabeth Sellers, Allison Dart, Garry Shen, Davinder Jassal and Nathan Nickel helped with interpretation of the data and revised the manuscript for important intellectual content. Dan Chateau and Heather Prior performed statistical analysis, contributed to writing the methods and results sections, and revised the manuscript for important intellectual content. Vernon Dolinsky, Joseph Gordon and Todd Duhamel contributed to the study design and revised the manuscript for important intellectual content. Jonathan McGavock contributed to study conception, data collection, and the preparation and finalization of the manuscript. All of the authors gave final approval of the version to be published and agreed to act as guarantors of the work.
Funding: Laetitia Guillemette was supported by a Canadian Institutes of Health Research (CIHR) Frederick Banting and Charles Best Canada Graduate Scholarship - Doctoral Award (380269), a Fonds de recherche en santé - Québec doctoral training award (30884) and the University of Manitoba Graduate Enhancement of Tri-Council Stipends program. Jonathan McGavock holds grants from the CIHR, Diabetes Canada, the Heart and Stroke Foundation of Canada (HSFC), the Cosmopolitan Foundation and the Lawson Foundation. Todd Duhamel holds grants from the HSFC and the CIHR. Joseph Gordon is supported by the Natural Sciences and Engineering Research Council of Canada and the HSFC. Vernon Dolinsky is funded by grants from the $\mathrm{CIHR}$ and the HSFC, and he is the Allen Rouse Basic Scientist of the Manitoba Medical Services Foundation. Brandy Wicklow has received funding from $\mathrm{ClHR}$, Diabetes Canada, the Children's Hospital Research Institute of Manitoba (CHRIM) and the Lawson Foundation. Elizabeth Sellers has received funding from Diabetes Canada, CHRIM and the Public Health Agency of Canada for unrelated investigator-led studies. Allison Dart currently has research funding from CIHR, Research Manitoba and CHRIM. Nathan Nickel holds grants from the CIHR, DEVOTION and Research Manitoba. Dan Chateau holds grants from CIHR. Laetitia Guillemette, Brandy Wicklow, Elizabeth Sellers, Allison Dart, Garry Shen, Vernon Dolinsky, Joseph Gordon, Nathan Nickel, Todd Duhamel and Jonathan McGavock were also supported by a Research Manitoba grant to the DEVOTION research cluster. None of the cited agencies were involved in the design, conduct or approval of this manuscript.

Data sharing: The data used in this study were obtained from the Manitoba Population Research Data Repository, housed at the Manitoba Centre for Health Policy, University of Manitoba, and were derived from data provided by Manitoba Health and the Winnipeg Regional Health Authority. These data are owned by the data providers: Manitoba
Health, Seniors and Active Living, and Manitoba Families. Access to the data may be granted upon receiving approvals from the University of Manitoba Health Research Ethics Board and the Health Information Privacy Committee, along with permission from both data providers. The authors are unable to provide direct access to the data without approval from the committees and ethics board, as stated above. More information about access to these databases is available at http://umanitoba.ca/faculties/health_sciences/ medicine/units/chs/departmental_units/mchp/ resources/access.html

Acknowledgements: The authors acknowledge the Manitoba Centre for Health Policy for the use of data contained in the Manitoba Population Research Data Repository under project \#Laetitia_thesis 2016040 (HIPC \#2016/2017-06). The results and conclusions are those of the authors and no official endorsement by the Manitoba Centre for Health Policy, Manitoba Health or other data providers is intended or should be inferred. The final draft of the manuscript was sent to the data providers (the Health Information Privacy Committee [HIPC] and the Winnipeg Regional Health Authority) regarding confidentiality, privacy and consistency with the HIPC-approved protocol, but these agencies had no control over the results presented or the decision to submit the manuscript for publication. The authors are deeply indebted to Yao Nie and Kristine Kroeker for their logistical support in developing the analytical plan throughout the revisions of this manuscript. The authors also acknowledge the support provided by the Children's Hospital Research Institute of Manitoba, the Children's Hospital Research Foundation, and the Diabetes Research Envisioned and Accomplished in Manitoba Theme at the Children's Hospital Research Institute of Manitoba.

Accepted: May 7, 2020

Correspondence to: Jonathan McGavock, jmcgavock@chrim.ca 